\title{
Ertugliflozin and Sitagliptin Co-initiation in Patients with Type 2 Diabetes: The VERTIS SITA Randomized Study
}

\author{
Sam Miller · Tania Krumins · Haojin Zhou · Susan Huyck • \\ Jeremy Johnson • Gregory Golm • Steven G. Terra · James P. Mancuso • \\ Samuel S. Engel · Brett Lauring (D)
}

Received: November 16, 2017 / Published online: January 8, 2018

(c) The Author(s) 2018. This article is an open access publication

\begin{abstract}
Introduction: Ertugliflozin is an oral sodiumglucose cotransporter 2 inhibitor that is being developed to treat type 2 diabetes mellitus (T2DM). This study assessed the efficacy and safety of co-initiation of ertugliflozin and sitagliptin compared with placebo in patients with T2DM inadequately controlled on diet and exercise.
\end{abstract}

Enhanced content To view enhanced content for this article go to http://www.medengine.com/Redeem/ 590DF0601376F750.

Electronic supplementary material The online version of this article (https://doi.org/10.1007/s13300017-0358-0) contains supplementary material, which is available to authorized users.

S. Miller

SAM Clinical Research Center, San Antonio, TX, USA

T. Krumins · S. Huyck · J. Johnson · G. Golm •

S. S. Engel · B. Lauring $(\square)$

Merck \& Co., Inc., Kenilworth, NJ, USA

e-mail: brett_lauring@merck.com

H. Zhou

MSD R\&D (China) Co., Ltd., Beijing, China

S. G. Terra

Pfizer, Inc., Andover, MA, USA

J. P. Mancuso

Pfizer Inc., Groton, CT, USA
Methods: In this phase III, randomized, doubleblind, multicenter, placebo-controlled 26-week study (NCT02226003), patients with T2DM and glycated hemoglobin (HbA1c) $8.0-10.5 \%$ on diet/exercise were randomized 1:1:1 to ertugliflozin $5 \mathrm{mg}$ once daily (QD) and sitagliptin $100 \mathrm{mg}$ QD (E5/S100), ertugliflozin $15 \mathrm{mg}$ QD and sitagliptin $100 \mathrm{mg}$ QD (E15/S100), or placebo. The primary efficacy endpoint was the change from baseline in HbA1c at week 26 .

Results: The mean baseline HbA1c of the randomized patients $(n=291)$ was $8.9 \%$. At week 26, both ertugliflozin/sitagliptin treatments provided significant reductions from baseline in HbA1c compared with placebo [least squares mean HbA1c change (95\% confidence intervals) from baseline was $-0.4 \%(-0.7$, $-0.2), \quad-1.6 \% \quad(-1.8,-1.4)$, and $-1.7 \%$ $(-1.9,-1.5)$ for placebo, E5/S100, and E15/ S100, respectively]. At week 26, 8.3\%, 35.7\%, and $31.3 \%$ of patients receiving placebo, E5/ S100, and E15/S100, respectively, had HbA1c $<7.0 \%$. Significant reductions in fasting plasma glucose, 2-h post-prandial glucose, body weight, and systolic blood pressure were observed with both ertugliflozin/sitagliptin groups compared with placebo. The incidence of adverse events (AEs) was similar across the groups. The incidences of the pre-specified AEs of urinary tract infection, genital mycotic infection, symptomatic hypoglycemia, and hypovolemia were low and not meaningfully different across groups. 
Conclusion: Co-initiation of ertugliflozin with sitagliptin in patients with T2DM inadequately controlled on diet and exercise provided a clinically meaningful improvement in glycemic control over 26 weeks.

Clinical Trial Registration: Clinicaltrials.gov NCT02226003.

Keywords: Ertugliflozin; Glycemic control; SGLT2 inhibitor; Sitagliptin; Type 2 diabetes mellitus

\section{INTRODUCTION}

Type 2 diabetes mellitus (T2DM) is a progressive disease, and with time many patients will require intensification of therapy [1]. Current guidelines support the initiation of two antihyperglycemic agents (AHAs) simultaneously for the treatment of patients with poor glycemic control who are unlikely to achieve the glycated hemoglobin (HbA1c) goal with the addition of a single agent [2-4]. Frequently, the addition of AHAs is inappropriately delayed, causing patients to experience unnecessarily long exposure to hyperglycemia [5-9]. Therefore, even patients with mild or moderate hyperglycemia at diagnosis might be best treated with initial dual-combination therapy. In either case, combining AHAs with complementary mechanisms of action and a low risk of hypoglycemia is appropriate [10].

Ertugliflozin and sitagliptin is one AHA combination that can be considered for co-initiation. Ertugliflozin is a highly selective sodium-glucose cotransporter 2 (SGLT2) inhibitor that is being developed for the treatment of patients with T2DM [11, 12]. Acting independently of insulin, SGLT2 inhibitors improve glycemic control through the inhibition of renal glucose reabsorption. This leads to enhanced urinary glucose excretion that in turn results in reduced plasma glucose [13]. Sitagliptin, a dipeptidyl peptidase-4 (DPP-4) inhibitor, improves glycemic control by increasing circulating levels of the incretin hormones glucagon-like peptide 1 and gastric inhibitory polypeptide, which augment glucose-dependent insulin secretion by $\beta$ cells $[14,15]$.
In previous clinical studies, ertugliflozin used as a monotherapy [16], as an add on to metformin [17], as an add on to the combination of sitagliptin and metformin [18], and in combination with sitagliptin as an add on to metformin $[19,20]$ improved glycemic control, reduced body weight, and generally reduced blood pressure with a low incidence of hypoglycemia. In other studies, sitagliptin used as a monotherapy [21] or as part of a dual therapy with metformin [22] also provided clinically meaningful reductions in blood glucose with a low risk of hypoglycemia or weight gain.

This study-VERTIS SITA-was designed to evaluate the safety and efficacy of co-initiation of treatment with ertugliflozin [5 or $15 \mathrm{mg}$ daily (QD)] and sitagliptin (100 mg QD), compared with placebo alone, in patients with T2DM and inadequate glycemic control on diet and exercise.

\section{METHODS}

\section{Study Design}

Protocol MK-8835-017 was a randomized, double-blind, placebo-controlled, parallel-group phase III study (VERTIS SITA; ClinicalTrials.gov identifier: NCT02226003). The study consisted of a 2 -week placebo run-in period followed by a 26-week double-blind treatment period. Patients receiving AHA therapy at screening entered $\mathrm{a} \geq 8$-week AHA wash-off period before beginning the run-in period. There was a posttreatment contact 14 days after the last dose of study medication for assessment of adverse events (AEs).

Patients eligible for the study and with adequate compliance during the placebo run-in ( $\geq 80 \%$ based on pill count) were randomized 1:1:1 to ertugliflozin $5 \mathrm{mg}$ QD plus sitagliptin $100 \mathrm{mg}$ QD (E5/S100), ertugliflozin $15 \mathrm{mg}$ QD plus sitagliptin $100 \mathrm{mg}$ QD (E15/S100), or placebo. Randomization was performed using a central electronic randomization system (interactive voice response system/integrated web response system). Ertugliflozin and sitagliptin tablets were packaged identically relative to their matching placebos. Patients, investigators, 
study site and central laboratory personnel, and the sponsor remained blinded during the 26-week treatment period. Patients were to take study medications in the morning without regard to food.

Glycemic rescue therapy with open-label glimepiride was prescribed for patients who met progressively more stringent glycemic rescue criteria, which consisted of fasting plasma glucose (FPG) values consistently (repeat measurement performed within 7 days) $>270 \mathrm{mg} / \mathrm{dL}$ $(>15.0 \mathrm{mmol} / \mathrm{L})$ after randomization through week 6 ; $>240 \mathrm{mg} / \mathrm{dL} \quad(13.3 \mathrm{mmol} / \mathrm{L})$ after week 6 through week 12; > $200 \mathrm{mg} / \mathrm{dL}$ $(11.1 \mathrm{mmol} / \mathrm{L})$ after week 12 through week 26 [23]. Rescued patients continued with the same dose and regimen of their study medications.

Mixed meal tolerance testing (MMTT) was performed at baseline and week 26 (or at rescue/ discontinuation visits if appropriate). For the test, patients consumed a standard meal consisting of two nutrition bars and one nutrition drink ( $\sim 680 \mathrm{kcal} ; 111 \mathrm{~g}$ carbohydrate, $14 \mathrm{~g}$ fat, $26 \mathrm{~g}$ protein). For the week 26 visit, patients were instructed to take their double-blind study medication at the clinic $1 \mathrm{~h}$ before consuming the meal. Blood samples were taken before starting the meal (time 0) and at 30 and $120 \mathrm{~min}$ following the start of the meal. The 120-min timepoint was used to assess effects on 2-h PPG, whereas the 30-min timepoint was used for assessment of the insulinogenic index, which was based on C-peptide [24].

Laboratory assessments were performed at a central laboratory.

All procedures followed were in accordance with the ethical standards of the responsible committee on human experimentation (institutional and national) and with the Helsinki Declaration of 1964, as revised in 2013. It was conducted in accordance with the principles of good clinical practice and approved by the appropriate institutional review boards and regulatory agencies. Informed consent was obtained from all individual participants included in the study. The protocol and statistical analysis plan were developed by the sponsors in consultation with an external Scientific Advisory Committee.

\section{Patient Population}

Patients $\geq 18$ years of age with T2DM according to the American Diabetes Association guidelines [25] and inadequate glycemic control [HbA1c $\geq 8.0 \% \quad$ and $\quad \leq 10.5 \% \quad(\geq 64$ and $\leq 91 \mathrm{mmol} / \mathrm{mol}$ )] on diet and exercise alone for $\geq 8$ weeks prior to screening, and who met all other eligibility criteria, could be enrolled in the study and proceed directly into a 2-week single-blind placebo run-in period prior to randomization. Patients with $\mathrm{HbA1c} \geq 7.5 \%$ and $\leq 10.0 \% \quad(\geq 58$ and $\leq 86 \mathrm{mmol} / \mathrm{mol}) \quad$ on either a single allowed AHA or a low-dose combination of two allowed AHAs $(\leq 50 \%$ of the maximum labeled dose for each AHA) were eligible to enter $\mathrm{a} \geq 8$-week AHA wash-off period. Patients with HbA1c $\geq 8.0 \%$ and $\leq 10.5 \%$ [ $\geq 64$ and $\leq 91 \mathrm{mmol} / \mathrm{mol}]$ after wash-off and who met the other eligibility criteria could enter the placebo run-in. Allowed AHAs at the time of the screening visit were metformin, $\alpha$-glucosidase inhibitors, sulfonylureas, and glinides.

Exclusion criteria included: history of type 1 diabetes mellitus or ketoacidosis; treatment in the previous 12 weeks with insulin of any type or with an AHA other than those listed above; active obstructive uropathy or an indwelling urinary catheter; estimated glomerular filtration rate (eGFR) $<60 \mathrm{~mL} / \mathrm{min} / 1.73 \mathrm{~m}^{2} ; \quad \mathrm{FPG}$ $>270 \mathrm{mg} / \mathrm{dL}(>15 \mathrm{mmol} / \mathrm{L})$ prior to initiation of the placebo run-in and confirmed within 7 days.

\section{Efficacy Assessments}

The primary efficacy endpoint was change from baseline in HbA1c at week 26. Pre-specified key secondary efficacy endpoints evaluated at week 26 were change from baseline in FPG and 2-hour (h) postprandial glucose (PPG); the proportion of patients with $\mathrm{HbA} 1 \mathrm{c}<7.0 \%$ ( $<53 \mathrm{mmol} / \mathrm{mol})$; and change from baseline in body weight, systolic blood pressure (SBP), and diastolic blood pressure (DBP). Other efficacy endpoints evaluated at week 26 were change from baseline in homeostasis model assessment of $\beta$-cell function (HOMA- $\beta$ ) and insulinogenic index with fasting C-peptide; percentage of 
patients who received glycemic rescue therapy through week 26; and percentage of patients with $\mathrm{HbA} 1 \mathrm{c}<6.5 \%(48 \mathrm{mmol} / \mathrm{mol})$.

The 2-h PPG was assessed from the MMTT performed at weeks 0 and 26 or at rescue or discontinuation. Body weight was measured in duplicate with a standardized digital scale. Sitting blood pressure (BP) was measured in triplicate using an automated oscillometric BP measuring device. The C-peptide-based insulinogenic index was defined as the ratio of the increment of C-peptide to that of plasma glucose $30 \mathrm{~min}$ after the MMTT. HOMA- $\beta$ was calculated using FPG and fasting C-peptide levels using the calculator released by the University of Oxford in 2004 [26].

\section{Safety Assessments}

Safety assessments included the incidence of AEs, including AEs of special interest [symptomatic hypoglycemia (defined as episodes with clinical symptoms reported by the investigator as hypoglycemia; biochemical documentation not required), AEs associated with genital mycotic infection (analyzed by gender), urinary tract infection (UTI), and hypovolemia]. In addition, documented hypoglycemia (symptomatic and asymptomatic), defined as episodes with a glucose level $\leq 70 \mathrm{mg} / \mathrm{dL}(3.9 \mathrm{mmol} / \mathrm{L})$, were recorded. Pre-specified laboratory, electrocardiogram, and postural BP parameters were assessed, as well as changes over time in laboratory parameters fincluding eGFR and lipid panels [including low-density lipoprotein cholesterol (LDL-C) and high-density lipoprotein cholesterol (HDL-C)]\} and vital signs.

\section{Statistical Analyses}

Randomization of approximately 300 patients (100 patients per group, expected to yield an effective sample size of 87 per group at week 26) was estimated to provide $>99 \%$ power to detect a true difference of $1.0 \%$ in the mean change from baseline in HbA1c between a given ertugliflozin dose plus sitagliptin and placebo, based on a two-sided test at the $5 \%$ level of significance.
For multiplicity control, the primary and key secondary efficacy endpoints were tested in the following order: HbA1c, FPG, 2-h PPG, proportion of patients with HbA1c $<7.0 \%$ ( $<53 \mathrm{mmol} / \mathrm{mol})$, body weight, SBP, and DBP. For each endpoint, E15/S100 was tested vs. placebo, followed by E5/S100 vs. placebo. Each test was performed at the 0.05 level, and testing continued until a $P$ value $\geq 0.05$ was obtained.

Efficacy analyses included all randomized patients who took $\geq 1$ dose of the study drug and had $\geq 1$ measurement of the respective endpoint. Post-rescue efficacy data were treated as missing in all efficacy analyses. A longitudinal data analysis (LDA) model [27] was used to evaluate continuous efficacy endpoints, with fixed effects for treatment, AHA status at screening, baseline eGFR (continuous), time (categorical), and interaction of time by treatment with a constraint that the true mean at baseline is common to all treatment groups (which is valid due to randomization). Missing data at week 26 were handled implicitly by the model. Logistic regression was used to evaluate the proportion of patients with HbA1c $<7.0 \%$ $(<53 \mathrm{mmol} / \mathrm{mol})$ at week 26 , fitted with terms for treatment, AHA status at screening, baseline eGFR (continuous), and baseline HbA1c (continuous), with missing data imputed via multiple imputation using the LDA model described above. The percentage of patients rescued was summarized in each treatment group. Reduction in HbA1c from baseline at week 26 was assessed in the subgroups with baseline HbA1c $\leq$ or $>$ the median $[9.0 \%$ $(75 \mathrm{mmol} / \mathrm{mol})]$ using a repeated measures analysis of covariance model.

Safety analyses included all randomized treated patients. Data following initiation of glycemic rescue were included for the analysis of serious AEs (SAEs), deaths, and discontinuations due to AEs, and excluded for the other endpoints. $P$ values and $95 \%$ confidence intervals (CIs) for between-group differences in prespecified AEs of interest were computed using the Miettinen and Nurminen method [28]. Changes from baseline in LDL-C and HDL-C were assessed by a LDA model similar to that used for the primary endpoint. Changes from 
baseline in eGFR and other safety endpoints were summarized descriptively.

\section{RESULTS}

Patients were randomized at 94 centers across nine countries (Bulgaria, Croatia, Czech Republic, Estonia, Hungary, Serbia, Ukraine, the United Kingdom, and the United States). The study started on September 25, 2014 and the last patient completed the study on February 23, 2016.

\section{Patient Disposition and Baseline Characteristics}

In total, 291 patients were randomized and 254 $(87.3 \%)$ completed the study on study medication [90 (91.8\%), 88 (91.7\%), and $76(78.4 \%)$ in the E5/S100, E15/S100, and placebo groups, respectively; Fig. S1 in the Electronic supplementary material, ESM]. In the placebo group, a higher percentage of patients discontinued the study medication due to 'withdrawal by patient' and 'lost to follow-up.'

Baseline demographics were generally similar between treatment groups (Table 1); the placebo group contained a higher percentage of patients from North America compared with either ertugliflozin/sitagliptin group. Overall, patients had a mean HbA1c of $8.9 \%$ (74 mmol/mol), a mean body mass index of $32.2 \mathrm{~kg} / \mathrm{m}^{2}$, a mean eGFR of $90.7 \mathrm{~mL} / \mathrm{min} /$ $1.73 \mathrm{~m}^{2}$, and a mean T2DM duration of 6.3 years. At screening, $51.9 \%$ of patients were receiving AHA treatment and entered the $\geq 8$ week AHA wash-off period prior to randomization.

\section{Efficacy}

At week 26, significantly greater reductions from baseline in $\mathrm{HbA1c}$ were observed in the E5/S100 and E15/S100 groups compared with placebo (Table 2; Fig. 1a). The least squares (LS) mean HbA1c changes (95\% CI) from baseline to week 26 were $-0.4 \% \quad(-0.7,-0.2),-1.6 \%$ $(-1.8,-1.4)$, and $-1.7 \%(-1.9,-1.5)$ in the placebo, E5/S100, and E15/S100 groups, respectively. The placebo-adjusted LS mean changes for E5/S100 and E15/S100 were $-1.2 \%$ $(-1.5,-0.8)$ and $-1.2 \% \quad(-1.6,-0.9)$, respectively $(P<0.001$ for both comparisons).

Both the ertugliflozin/sitagliptin groups provided mean HbA1c reductions from baseline in patient subgroups analyzed by baseline HbA1c $\{\leq$ and $>$ median $[9.0 \%(75 \mathrm{mmol} / \mathrm{mol})]$ baseline $\mathrm{HbA1c}$; smaller changes were observed in all treatment groups in the lower baseline subgroup and greater changes were observed in the subgroup with higher baseline HbA1c (Table S1 in the ESM).

A higher percentage of the patients treated with $\mathrm{E} 5 / \mathrm{S} 100$ and $\mathrm{E} 15 / \mathrm{S} 100$ had $\mathrm{HbA1c}<7.0 \%$ $(<53 \mathrm{mmol} / \mathrm{mol})$ at week 26 compared with the placebo group (Table 3 ). The odds of having HbA1c $<7.0 \% \quad(<53 \mathrm{mmol} / \mathrm{mol})$ at week 26 were significantly greater in the E5/S100 and E15/S100 groups than in the placebo group (both $P<0.001$ ) (Table 3). Similarly, the odds of having $\mathrm{HbA} 1 \mathrm{c}<6.5 \%(<48 \mathrm{mmol} / \mathrm{mol})$ at week 26 were greater in the E5/S100 and E15/S100 groups than in the placebo group (Table S2 in the ESM).

Significantly greater reductions from baseline were observed at week 26 for the E5/S100 and E15/S100 groups in the key secondary endpoints of FPG, 2-h PPG, body weight, and SBP compared with placebo (Table 3; Fig. 1b, c). Placebo-adjusted reductions in DBP were observed for E5/S100 and E15/S100, but were not statistically significant (Table 3).

Fewer of the patients treated with E5/S100 received glycemic rescue medication through week 26 (6.1\%) compared with placebo $(32.0 \%$; Table S2 in the ESM). None of the patients treated with E15/S100 required glycemic rescue medication during the study.

LS mean increases from baseline in HOMA- $\beta$ (\%) at week 26 were greater in the E5/S100 and E15/S100 groups than in the placebo group $(P<0.001$ for both comparisons; Table S2 in the ESM). A trend toward an improvement in C-peptide-based insulinogenic index at week 26 was observed in the E5/S100 and E15/S100 groups relative to the placebo group (Table S2 in the ESM). 
Table 1 Baseline demographics and disease characteristics

\begin{tabular}{|c|c|c|c|c|}
\hline & $\begin{array}{l}\text { Placebo } \\
(n=97)\end{array}$ & $\begin{array}{l}\mathrm{E} 5 / \mathrm{S} 100 \\
(n=98)\end{array}$ & $\begin{array}{l}\mathrm{E} 15 / \mathrm{S} 100 \\
(n=96)\end{array}$ & $\begin{array}{l}\text { Total } \\
(n=291)\end{array}$ \\
\hline Age, years & $54.3(10.3)$ & $56.4(9.3)$ & $56.1(10.1)$ & $55.6(10.0)$ \\
\hline Male, $n(\%)$ & $57(58.8)$ & $57(58.2)$ & $53(55.2)$ & $167(57.4)$ \\
\hline \multicolumn{5}{|l|}{ Race/ethnicity, $n$ (\%) } \\
\hline White & $90(92.8)$ & $92(93.9)$ & $81(84.4)$ & $263(90.4)$ \\
\hline Black or African American & $4(4.1)$ & $2(2.0)$ & $7(7.3)$ & $13(4.5)$ \\
\hline American Indian or Alaska Native & $2(2.1)$ & $4(4.1)$ & $6(6.3)$ & $12(4.1)$ \\
\hline Multiple & $1(1.0)$ & $0(0.0)$ & $1(1.0)$ & $2(0.7)$ \\
\hline Native Hawaiian or other Pacific Islander & $0(0.0)$ & $0(0.0)$ & $1(1.0)$ & $1(0.3)$ \\
\hline Hispanic or Latino & $37(38.1)$ & $34(34.7)$ & $34(35.4)$ & $105(36.1)$ \\
\hline \multicolumn{5}{|l|}{ Region, $n(\%)$} \\
\hline North America (excl. Central America) & $57(58.8)$ & $41(41.8)$ & $45(46.9)$ & $143(49.1)$ \\
\hline Europe (incl. Russia) & $40(41.2)$ & $57(58.2)$ & $51(53.1)$ & $148(50.9)$ \\
\hline Body weight, $\mathrm{kg}$ & $95.0(20.5)$ & $90.8(20.7)$ & $91.2(22.5)$ & $92.3(21.3)$ \\
\hline BMI, $\mathrm{kg} / \mathrm{m}^{2}$ & $32.7(6.2)$ & $32.0(6.3)$ & $32.1(5.8)$ & $32.2(6.1)$ \\
\hline Duration of T2DM, years & $6.8(6.5)$ & $5.7(5.0)$ & $6.5(6.5)$ & $6.3(6.05)$ \\
\hline HbAlc, \% & $9.0(0.9)$ & $8.9(0.9)$ & $9.0(0.9)$ & $8.9(0.9)$ \\
\hline $\mathrm{HbAlc}, \mathrm{mmol} / \mathrm{mol}$ & $74.3(9.4)$ & $73.7(9.5)$ & $74.6(9.5)$ & $74.2(9.4)$ \\
\hline $\mathrm{FPG}, \mathrm{mg} / \mathrm{dL}$ & $207.5(44.9)$ & $198.0(47.7)$ & $187.7(46.7)$ & $197.8(47.0)$ \\
\hline $\mathrm{FPG}, \mathrm{mmol} / \mathrm{L}$ & $11.5(2.5)$ & $11.0(2.6)$ & $10.4(2.6)$ & $11.0(2.3)$ \\
\hline \multicolumn{5}{|l|}{ Background AHA therapy status at screening, $n$ (\%) } \\
\hline Currently on AHA therapy & $50(51.5)$ & $49(50.0)$ & $52(54.2)$ & $151(51.9)$ \\
\hline Not currently on AHA therapy, previously treated & $16(16.5)$ & $15(15.3)$ & $11(11.5)$ & $42(14.4)$ \\
\hline Never treated & $31(32.0)$ & $34(34.7)$ & $33(34.4)$ & $98(33.7)$ \\
\hline $\mathrm{eGFR}, \mathrm{mL} / \mathrm{min} / 1.73 \mathrm{~m}^{2}$ & $92.6(21.6)$ & $90.0(17.2)$ & $89.5(18.1)$ & $90.7(19.0)$ \\
\hline Serum creatinine, $\mathrm{mg} / \mathrm{dL}$ & $0.8(0.2)$ & $0.8(0.1)$ & $0.8(0.2)$ & - \\
\hline
\end{tabular}

Data are presented as mean ( \pm standard deviation) unless otherwise stated

$H b A 1 c$ glycated hemoglobin, $A H A$ antihyperglycemic agents, $B M I$ body mass index, $E$ ertugliflozin, eGFR estimated glomerular filtration rate, $F P G$ fasting plasma glucose, $S$ sitagliptin, $T 2 D M$ type 2 diabetes mellitus 
Table 2 Change in glycated hemoglobin (HbAlc) from baseline at week 26

\begin{tabular}{|c|c|c|c|}
\hline & Placebo & $\mathrm{E} 5 / \mathrm{S} 100$ & E15/S100 \\
\hline Baseline & $n=96$ & $n=98$ & $n=96$ \\
\hline Mean (SD), \% & $9.0(0.9)$ & $8.9(0.9)$ & $9.0(0.9)$ \\
\hline Mean (SD), $\mathrm{mmol} / \mathrm{mol}$ & $74.3(9.4)$ & $73.7(9.5)$ & $74.6(9.5)$ \\
\hline Week 26 & $n=49$ & $n=85$ & $n=82$ \\
\hline Mean (SD), \% & $8.0(1.1)$ & $7.2(1.0)$ & $7.3(1.0)$ \\
\hline Mean (SD), $\mathrm{mmol} / \mathrm{mol}$ & $64.0(12.3)$ & $54.8(10.9)$ & $56.0(11.0)$ \\
\hline Change from baseline at week 26 & $n=96$ & $n=98$ & $n=96$ \\
\hline Mean (SD), \% & $-0.8(1.1)$ & $-1.7(0.9)$ & $-1.7(1.1)$ \\
\hline Mean $(\mathrm{SD}), \mathrm{mmol} / \mathrm{mol}$ & $-8.5(11.6)$ & $-18.4(9.8)$ & $-18.3(11.7)$ \\
\hline LS mean (95\% CI), \% & $-0.4(-0.7,-0.2)$ & $-1.6(-1.8,-1.4)$ & $-1.7(-1.9,-1.5)$ \\
\hline LS mean $(95 \% \mathrm{CI}), \mathrm{mmol} / \mathrm{mol}$ & $-4.8(-7.6,-2.1)$ & $-17.5(-19.9,-15.1)$ & $-18.4(-20.8,-16.0)$ \\
\hline \multicolumn{4}{|l|}{ Pairwise comparison vs. placebo } \\
\hline Difference in LS means (95\% CI), \% & - & $-1.2(-1.5,-0.8)$ & $-1.2(-1.6,-0.9)$ \\
\hline $\begin{array}{l}\text { Difference in LS means (95\% CI), } \\
\mathrm{mmol} / \mathrm{mol}\end{array}$ & - & $-12.7(-16.2,-9.1)$ & $-13.5(-17.1,-10.0)$ \\
\hline$P$ value & - & $<0.001$ & $<0.001$ \\
\hline
\end{tabular}

$C I$ confidence interval, $E$ ertugliflozin, $L S$ least squares, $S$ sitagliptin, $S D$ standard deviation

\section{Safety}

The percentage of patients with one or more $\mathrm{AE}$ was similar across the groups (Table 4). The incidences of SAEs and AEs leading to discontinuation were low and not meaningfully different across treatment groups. No deaths were reported. The incidence of drug-related AEs was slightly higher in the E15/S100 group than in the placebo group and was similar between the E5/S100 and placebo groups. The excess of drugrelated AEs in the E15/S100 group was not due to any particular AE or group of AEs.

In male patients, the incidence of genital mycotic infections was 0\% (placebo), 5.3\% (E5/ S100), and 1.9\% (E15/S100) (Table 4). The incidence of genital mycotic infections among female patients was similar across treatment groups (Table 4). The incidences of UTIs, hypovolemia, and symptomatic hypoglycemia were generally low and not notably different between the treatment groups (Table 4). The observed incidence of documented hypoglycemia in the E5/S100 group (6.1\%) was higher than that in the E15/S100 (3.1\%) and placebo (1.0\%) groups. Two events of severe hypoglycemia (requiring nonmedical assistance) were reported in the E15/S100 treatment group. No events of severe hypoglycemia requiring medical assistance were reported in any treatment group.

At week 6 (first post-randomization assessment), modest reductions in eGFR were observed in all groups. Values of eGFR returned to or near baseline by week 26 in all treatment groups (Fig. 2). Overall, 2.3\%, 2.1\%, and $2.1 \%$ of patients in the placebo, E5/S100, and E15/S100 groups, respectively, had a decrease from baseline of $>30 \%$ in eGFR on at least one occasion. 

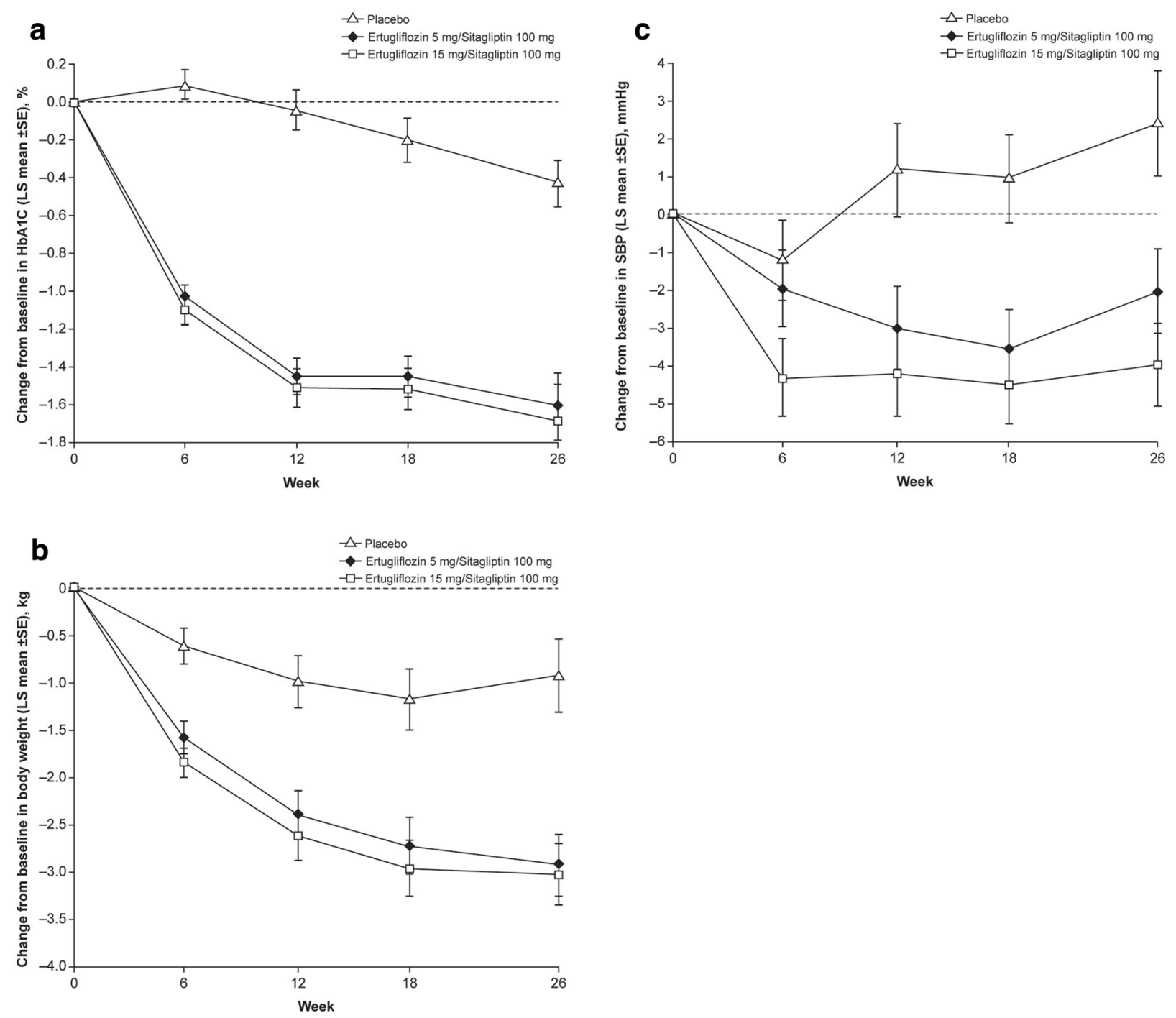

Fig. 1 Change over time in a glycated hemoglobin (HbAlc), b body weight, and c systolic blood pressure $(\mathrm{SBP})^{\mathrm{a}}$. $L S$ least squares, $S E$ standard error. ${ }^{\text {a }}$ Relevant $p$ values are provided in Tables 2 and 3

One patient $(1.1 \%)$ in the placebo group had a decrease of $>50 \%$ in eGFR from baseline. No patients discontinued the study medication because they met the protocol-specified discontinuation threshold for eGFR [value consistently (repeated within 7 days) $<50 \mathrm{~mL} / \mathrm{min} /$ $1.73 \mathrm{~m}^{2}$ ].

Small increases from baseline in LDL-C at week 26 were observed in all groups, and increases were greater in the E5/S100 and E15/ S100 groups vs. the placebo group (LS mean percent change from baseline: $2.9 \%[-3.5,9.2]$, $10.6 \%[4.9,16.3]$, and $10.1 \%[4.4,15.8]$ for placebo, E5/S100, and E15/S100, respectively; Table S3 in the ESM). Small increases from baseline in HDL-C at week 26 were also observed across all groups; these were greater in the E5/S100 and E15/S100 groups vs. the placebo group (LS mean percent change from baseline: $3.6 \%$ [- 0.3 . 7.5], 9.3\% [5.8, 12.7], and $8.2 \%[4.8,11.6]$ for placebo, E5/S100, and E15/ S100, respectively; Table S3 in the ESM).

Mean (SD) changes in hemoglobin from baseline at week 26 were $-0.2 \mathrm{~g} / \mathrm{dL}(0.7)$, $0.5 \mathrm{~g} / \mathrm{dL}(0.9)$, and $0.5 \mathrm{~g} / \mathrm{dL}(0.8)$ for placebo, E5/S100, and E15/S100, respectively. Mean (SD) 


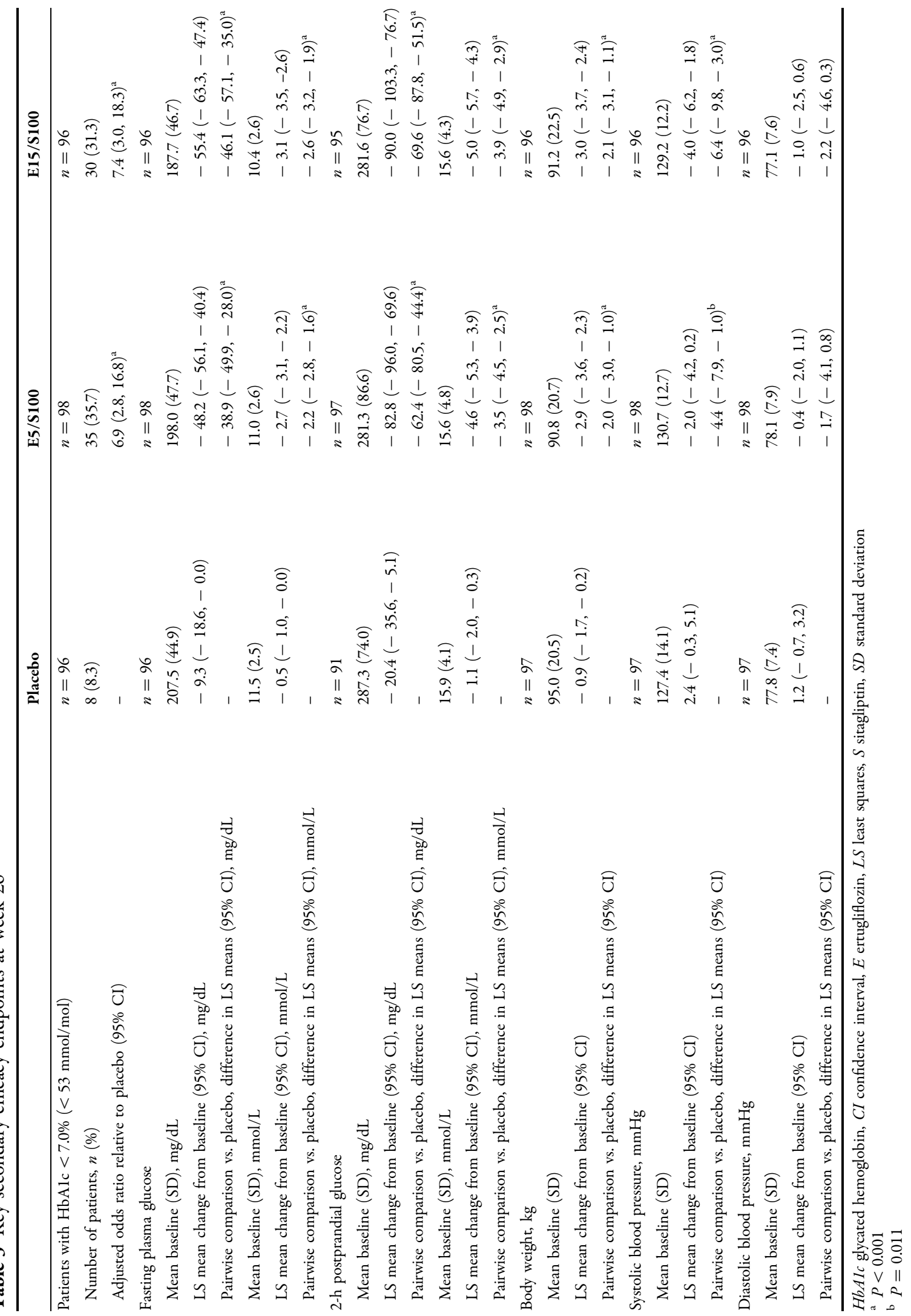


Table 4 Summary of overall safety and pre-specified adverse events (AEs)

\begin{tabular}{|c|c|c|c|}
\hline Number of patients, $n(\%)$ & $\begin{array}{l}\text { Placebo } \\
(n=97)\end{array}$ & $\begin{array}{l}\mathrm{E} 5 / \mathrm{S} 100 \\
(n=98)\end{array}$ & $\begin{array}{l}\mathrm{E} 15 / \mathrm{S} 100 \\
(n=96)\end{array}$ \\
\hline \multicolumn{4}{|l|}{ Overall safety } \\
\hline One or more AEs (ER) & $41(42.3)$ & $44(44.9)$ & $43(44.8)$ \\
\hline AEs related to study drug $(E R)^{a}$ & $8(8.2)$ & $9(9.2)$ & $13(13.5)$ \\
\hline One or more SAEs (IR) & $5(5.2)$ & $2(2.0)$ & $3(3.1)$ \\
\hline Serious AEs related to study drug (IR) & $0(0)$ & $0(0)$ & $0(0)$ \\
\hline Deaths (IR) & $0(0)$ & $0(0)$ & $0(0)$ \\
\hline AEs leading to discontinuation (IR) & $3(3.1)$ & $2(2.0)$ & $2(2.1)$ \\
\hline \multicolumn{4}{|l|}{ Pre-specified AEs $(E R)^{b}$} \\
\hline Genital mycotic infection (men) & $0(0.0)$ & $3(5.3)$ & $1(1.9)$ \\
\hline Genital mycotic infection (women) & $2(5.0)$ & $2(4.9)$ & $3(7.0)$ \\
\hline Urinary tract infection & $5(5.2)$ & $8(8.2)$ & $3(3.1)$ \\
\hline Symptomatic hypoglycemia ${ }^{c}$ & $1(1.0)$ & $3(3.1)$ & $3(3.1)$ \\
\hline Hypovolemia & $1(1.0)$ & $2(2.0)$ & $2(2.1)$ \\
\hline
\end{tabular}

$A E$ adverse event, $E$ ertugliflozin, $E R$ analysis excludes events occurring after the initiation of rescue medication, $I R$ analysis includes events occurring after the initiation of rescue medication, $S$ sitagliptin, $S A E$ serious adverse event

a AEs reported by the investigator

b Pre-specified AEs were specified for inferential testing without multiplicity control; no $P$ values for comparisons to placebo were $<0.05$

${ }^{c}$ Event with clinical symptoms reported by the investigator as hypoglycemia (concurrent finger stick glucose not required)

hematocrit changes from baseline at week 26 were $-0.2 \%$ (2.3), $2.2 \%$ (2.8), and $2.5 \%$ (2.5) for placebo, E5/S100, and E15/S100, respectively.

Neither ketoacidosis nor pancreatitis was observed in any of the treatment groups. There was one adjudication-confirmed fracture event (multiple fractures sustained in a motor vehicle accident) in one patient in the placebo group. There were no cases of amputation in this study.

\section{DISCUSSION}

In this phase III, randomized, double-blind, multicenter, placebo-controlled clinical study (VERTIS SITA), significant improvements in glycemic control were observed after 26 weeks of treatment with the combination of ertugliflozin and sitagliptin compared with placebo alone in patients with T2DM and inadequate glycemic control on diet and exercise. For E5/S100 and
E15/S100, large reductions in HbA1c were observed as early as the first post-randomization visit at week 6 , with additional reductions seen through week 26 . Significantly more patients treated with E5/S100 and E15/S100 met the HbA1c target of $<7.0 \%(<53 \mathrm{mmol} / \mathrm{mol})$ [29] compared with the placebo group at week 26 . In addition, statistically significant, clinically meaningful reductions in FPG, 2-h PPG, body weight, and SBP were seen among patients who received ertugliflozin plus sitagliptin vs. placebo. The significant reductions in SBP observed with ertugliflozin and sitagliptin treatment were noteworthy, as approximately 50\% of patients were receiving BP medication at baseline and SBP was generally well controlled (mean baseline SBP $\sim 130 \mathrm{mmHg}$ ).

The efficacy of combination therapy with ertugliflozin and sitagliptin observed in this study is consistent with that observed in other 

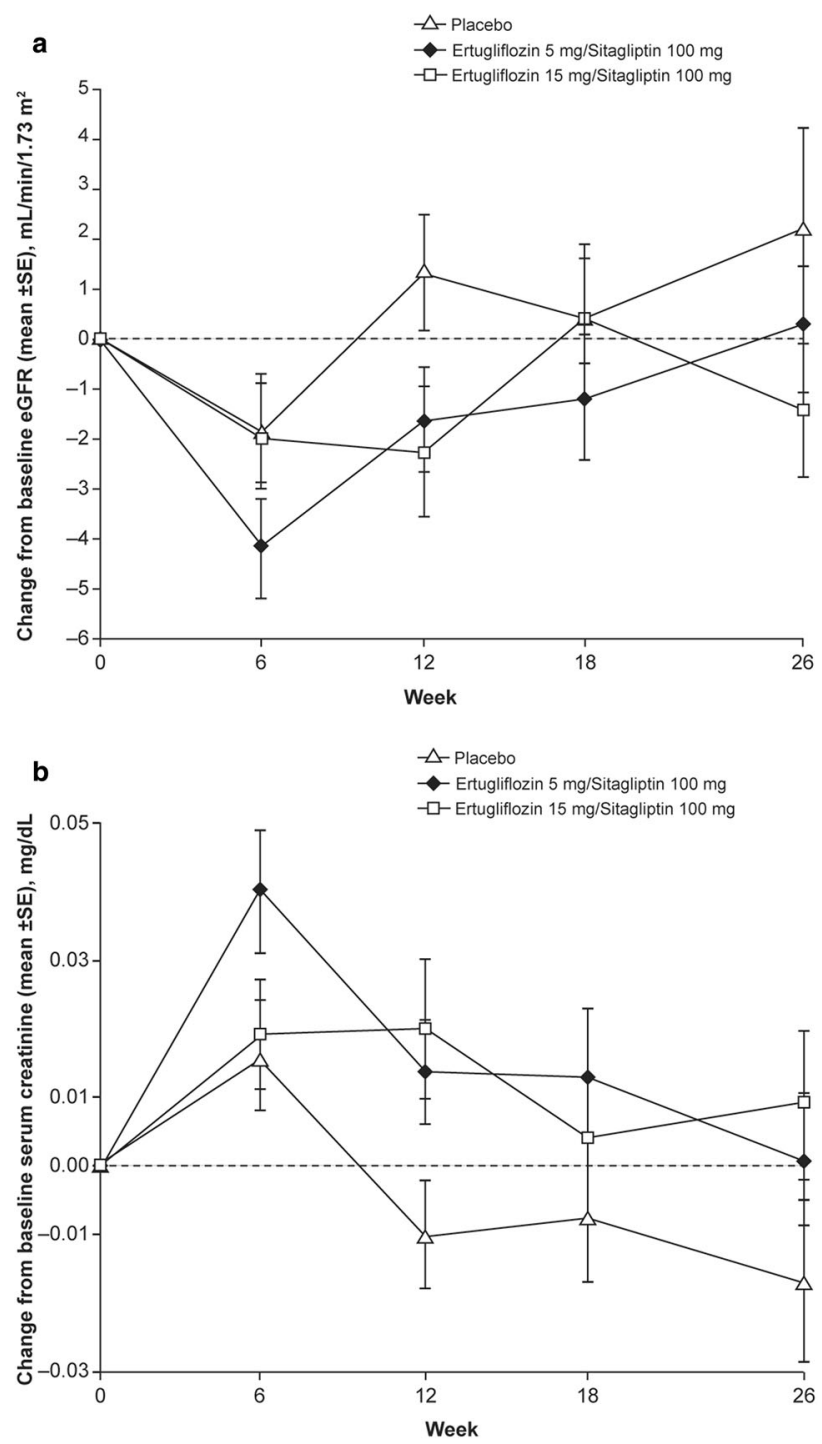

Fig. 2 a Mean change from baseline in estimated glomerular filtration rate (eGFR) $\left(\mathrm{mL} / \mathrm{min} / 1.73 \mathrm{~m}^{2}\right)$ through week 26 . b Mean change from baseline in serum creatinine $(\mathrm{mg} / \mathrm{dL})$ through week $26 . S E$ standard error

studies in the VERTIS program $[18,19]$. In the VERTIS FACTORIAL study, adding the combination of ertugliflozin (5 or $15 \mathrm{mg}$ ) and sitagliptin to the treatment of patients on metformin reduced $\mathrm{HbA} 1 \mathrm{c}$ by $1.5 \%$ from a baseline of $8.6 \%(70 \mathrm{mmol} / \mathrm{mol})$, which was superior to the effect of either agent alone $[19,20]$. In the present study, patients were not receiving background AHAs, and the mean baseline HbA1c of $8.9 \%$ was slightly higher than that in VERTIS FACTORIAL; the observed LS mean reductions from baseline in $\mathrm{HbA1c}$ for the E5/S100 and E15/S100 groups were slightly larger than those observed in the VERTIS FACTORIAL study $(1.5 \%$ for both combination therapy groups). Furthermore, the significant reductions from baseline in body weight and SBP observed in the study reported here are consistent with the reductions observed after treatment with ertugliflozin alone or in combination with other AHAs across the VERTIS program $[16,17,19]$. In another study assessing 
co-initiation of a DPP-4 inhibitor with an SGLT2 inhibitor vs. each individual treatment in patients with T2DM inadequately controlled on diet and exercise, co-initiation had beneficial effects on glycemic control and body weight reduction, with modest reductions in blood pressure also observed [30].

The two doses of ertugliflozin assessed together with sitagliptin in this study were selected for phase III evaluation because dose-response modeling from phase II studies indicated that ertugliflozin 5 and $15 \mathrm{mg}$ would provide 80\% and $90 \%$ of maximal efficacy, respectively $[11,12]$. The current study was not powered to detect differences between ertugliflozin doses, and co-initiation studies may not be the most appropriate approach to discern differences between drug doses. However, while the differences in efficacy between the two ertugliflozin doses were modest, point estimates for E15/ S100 consistently demonstrated slightly greater efficacy than for E5/S100 with regard to reductions in HbA1c, FPG, 2-h PPG, body weight, and SBP. Further, no patient in the E15/S100 group required the addition of glycemic rescue medication during the study, as compared with $6.1 \%$ in the E5/S100 group. These results are consistent with similarly powered studies in which, as a monotherapy [16], as an add on to metformin [17], and as an add on to metformin and sitagliptin [18], the $15 \mathrm{mg}$ ertugliflozin dose provided $0.2 \%, 0.2 \%$, and $0.1 \%$ greater reductions in HbA1c, respectively, compared with the $5 \mathrm{mg}$ dose, suggesting the higher dose is generally associated with greater SGLT2 inhibition and efficacy.

The magnitude of the HbA1c decrease in the placebo group $(-0.4 \%)$ may be explained by the high mean baseline HbA1c levels in the study population [approximately 9\% ( $75 \mathrm{mmol} / \mathrm{mol})]$, leading to a larger trial effect than typically observed. Of note, the placebo response was larger $(-1.0 \%)$ for those with baseline HbA1c values greater than the median [>9.0\% (> $75 \mathrm{mmol} / \mathrm{mol})]$ compared to those with baseline $\mathrm{HbA1c}$ values below the median $(0.0 \%)$.

Recent small mechanistic studies have suggested that SGLT2 inhibition may lead to increased insulin sensitivity and improved $\beta$ - cell function [31, 32]. In the current study, ertugliflozin plus sitagliptin was associated with improvements in HOMA- $\beta$, a marker of $\beta$-cell function. The underlying mechanism is unknown, but it could be a secondary response to reduced glucotoxicity resulting from enhanced urinary elimination of glucose. Similar effects have been observed in other ertugliflozin studies $[16,18]$.

Although metformin is the established firstline therapy for T2DM unless contraindicated or not tolerated [1], patients who are not on pharmacotherapy and present with high baseline $\mathrm{HbA1c}$, as in this study [mean baseline HbA1c of approximately $9 \%(75 \mathrm{mmol} / \mathrm{mol})]$, may be less likely than patients with lower HbA1c to achieve the treatment goal with the initiation of monotherapy. In this study, $31.3-35.7 \%$ of patients treated with ertugliflozin/sitagliptin achieved HbA1c $<7.0 \%$ $(<53 \mathrm{mmol} / \mathrm{mol})$ by the end of the study, compared with $8.3 \%$ of patients in the placebo group.

Combining ertugliflozin with sitagliptin was generally well tolerated over 26 weeks. No meaningful differences were seen in the incidences of pre-specified AEs of interest [symptomatic hypoglycemia, UTIs, genital mycotic infections (male or female), or hypovolemia] between groups. SGLT2 inhibitors have been linked to transient decreases in eGFR, likely hemodynamically mediated [33, 34]. Modest, transient decreases in mean eGFR were observed in this study in all groups; however, mean eGFRs returned to or near baseline levels at week 26 . The pattern of changes in eGFR from baseline was consistent with prior observations with SGLT2 inhibitors [35]. These transient reductions in eGFR may reflect an acute osmotic diuretic effect along with effects on tubuloglomerular feedback, resulting in afferent arteriolar vasoconstriction [33, 34]. Recent evidence suggests that SGLT2 inhibitors may have long-term beneficial effects on renal outcomes, which is probably the result of several direct and indirect effects on the kidney through tubuloglomerular feedback and improvements in hyperglycemia, hypertension, obesity, and hyperuricemia [36, 37]. There were no events of 
pancreatitis, ketoacidosis, amputation, or fracture in ertugliflozin/sitagliptin-treated patients.

This study has certain limitations. This was a placebo-controlled study of the combination of ertugliflozin and sitagliptin in patients not adequately controlled on diet and exercise. As such, there is no direct comparison to metformin, which is the recommended first-line therapy in most patients with T2DM [1]. Similarly, not all patients in the present study were drug-naïve, and around 50\% washed off prior therapy prior to the placebo run-in and randomization for this study.

\section{CONCLUSION}

In summary, in patients with T2DM who had inadequate glycemic control with diet and exercise alone, co-initiation of ertugliflozin and sitagliptin provided clinically meaningful improvements in glycemic control and reduced body weight and SBP relative to placebo. Combination treatment was generally well tolerated, without a meaningful difference in symptomatic hypoglycemia, UTIs, hypovolemia, or genital mycotic infections relative to placebo.

\section{ACKNOWLEDGEMENTS}

The authors wish to thank the investigators, staff and participants in the VERTIS SITA study (protocol MK-8835-017). Data from this study were previously presented at the 77th Scientific Sessions of the American Diabetes Association (San Diego, USA) and the 53rd Annual Meeting of the European Association for the Study of Diabetes (Lisbon, Portugal).

Funding. Merck Sharp \& Dohme Corp., a subsidiary of Merck \& Co., Inc., Kenilworth, NJ, USA, in collaboration with Pfizer Inc., provided financial support for this study. Merck Sharp \& Dohme Corp., a subsidiary of Merck \& Co, Inc., Kenilworth, NJ, USA, provided funding for article processing charges for this publication. All authors had full access to all of the data in this study and take complete responsibility for the integrity of the data and accuracy of the data analysis.

Medical Writing and/or Editorial Assistance. Medical writing support, including assisting authors with the development of the initial draft and the incorporation of comments, fact checking, referencing and proofreading, was provided by Elien Moës, PhD, ISMPP CMPP ${ }^{\mathrm{TM}}$, Camille Bonomelli, PhD and Faye Gould, PhD, ISMPP CMPP ${ }^{\mathrm{TM}}$, and editorial support, including figure preparation, formatting, and submission was provided by Nicola Jenkins, MA (all of Scion, London, UK). This assistance was funded by Merck Sharp \& Dohme Corp., a subsidiary of Merck \& Co., Inc., Kenilworth, NJ, USA. Ed O'Neill, PhD (Merck \& Co., Inc., Kenilworth, NJ, USA) provided additional editing. The sponsor was involved in the study design, collection, analysis and interpretation of data, as well as the data checking of information provided in the manuscript. However, ultimate responsibility for opinions, conclusions, and data interpretation lies with the authors.

Authorship. All named authors meet the International Committee of Medical Journal Editors (ICMJE) criteria for authorship for this manuscript, take responsibility for the integrity of the work as a whole, and have given final approval to the version to be published.

Author Contributions. Brett Lauring, Jeremy Johnson, Gregory Golm, James P. Mancuso, Steven G. Terra, and Samuel S. Engel, contributed to the conception, design, or planning of the study. Brett Lauring, Sam Miller, Tania Krumins and Jeremy Johnson contributed to the acquisition of the data. Brett Lauring, Haojin Zhou, Susan Huyck, Gregory Golm, and James P. Mancuso participated in the analysis of the data. Brett Lauring, Sam Miller, Haojin Zhou, Susan Huyck, Jeremy Johnson, Gregory Golm, James P. Mancuso, Steven G. Terra, and Samuel S. Engel contributed to the interpretation of the results. Authors are responsible for the work described in this paper. All authors reviewed the manuscript for important intellectual content and provided final approval of 
the version to be published. All authors agree to be accountable for all aspects of the work in ensuring that questions related to the accuracy or integrity of any part of the work are appropriately investigated and resolved.

Disclosures. Sam Miller has received research support from Merck \& Co., Inc., Kenilworth, NJ, USA; other support from AstraZeneca, Bristol-Myers Squibb Company, Gelesis, Lilly, Pfizer, Boehringer Ingelheim, Novartis, Johnson and Johnson, Novo Nordisk, Tadeka, Amgen. Brett Lauring is employed by Merck Sharp \& Dohme Corp., a subsidiary of Merck \& Co., Inc., Kenilworth, NJ, USA, and may own stock and/or hold stock options in the company. Tania Krumins is employed by Merck Sharp \& Dohme Corp., a subsidiary of Merck \& Co., Inc., Kenilworth, NJ, USA, and may own stock and/or hold stock options in the company. Jeremy Johnson is employed by Merck Sharp \& Dohme Corp., a subsidiary of Merck \& Co., Inc., Kenilworth, NJ, USA, and may own stock and/or hold stock options in the company. Susan Huyck is employed by Merck Sharp \& Dohme Corp., a subsidiary of Merck \& Co., Inc., Kenilworth, NJ, USA, and may own stock and/or hold stock options in the company. Gregory Golm is employed by Merck Sharp \& Dohme Corp., a subsidiary of Merck \& Co., Inc., Kenilworth, NJ, USA, and may own stock and/or hold stock options in the company. Samuel S. Engel is employed by Merck Sharp \& Dohme Corp., a subsidiary of Merck \& Co., Inc., Kenilworth, NJ, USA, and may own stock and/or hold stock options in the company. Haojin Zhou is employed by MSD R\&D (China) Co., Ltd., a subsidiary of Merck \& Co., Inc., Kenilworth, NJ, USA, and may own stock and/or hold stock options in the company. Steven G. Terra is employed by and is a shareholder in Pfizer Inc. James P. Mancuso is employed by and is a shareholder in Pfizer Inc.

Compliance with Ethics Guidelines. All procedures followed were in accordance with the ethical standards of the responsible committee on human experimentation (institutional and national) and with the Helsinki Declaration of 1964, as revised in 2013. It was conducted in accordance with the principles of good clinical practice and approved by the appropriate institutional review boards and regulatory agencies. Informed consent was obtained from all individual participants included in the study. The protocol and statistical analysis plan were developed by the sponsors in consultation with an external Scientific Advisory Committee.

Data Availability. Merck \& Co., Inc.'s data sharing policy, including restrictions, is available at http://engagezone.merck.com/ds documentation.php. Requests for access to the study data can be submitted through the EngageZone site or via email to dataaccess@merck.com.

Open Access. This article is distributed under the terms of the Creative Commons Attribution-NonCommercial 4.0 International License (http://creativecommons.org/licenses/ by-nc/4.0/), which permits any noncommercial use, distribution, and reproduction in any medium, provided you give appropriate credit to the original author(s) and the source, provide a link to the Creative Commons license, and indicate if changes were made.

\section{REFERENCES}

1. American Diabetes Association. 8. Pharmacologic approaches to glycemic treatment. Diabetes Care. 2017;40:S64-74.

2. Inzucchi SE, Bergenstal RM, Buse JB, Diamant M, Ferrannini E, Nauck $M$, et al. Management of hyperglycemia in type 2 diabetes, 2015: a patientcentered approach: update to a position statement of the American Diabetes Association and the European Association for the Study of Diabetes. Diabetes Care. 2015;38:140-9.

3. Garber AJ, Abrahamson MJ, Barzilay JI, Blonde L, Bloomgarden ZT, Bush MA, et al. Consensus statement by the American Association of Clinical Endocrinologists and American College of Endocrinology on the comprehensive type 2 diabetes management algorithm-2017 executive summary. Endocr Pract. 2017;23:207-38. 
4. Harper W, Clement M, Goldenberg R, Hanna A, Main A, Retnakaran R, et al. Pharmacologic management of type 2 diabetes. Can J Diabetes. 2013;37:S61-8.

5. Korytkowski MT, Brooks M, Lombardero M, DeAlmeida D, Kanter J, Magaji V, et al. Use of an electronic medical record (EMR) to identify glycemic intensification strategies in type 2 diabetes. J Diabetes Sci Technol. 2015;9:593-601.

6. Khunti K, Wolden ML, Thorsted BL, Andersen M, Davies MJ. Clinical inertia in people with type 2 diabetes: a retrospective cohort study of more than 80,000 people. Diabetes Care. 2013;36:3411-7.

7. Fu AZ, Qiu Y, Davies MJ, Radican L, Engel SS. Treatment intensification in patients with type 2 diabetes who failed metformin monotherapy. Diabetes Obes Metab. 2011;13:765-9.

8. Olansky L, Reasner C, Seck TL, Williams-Herman DE, Chen M, Terranella L, et al. A treatment strategy implementing combination therapy with sitagliptin and metformin results in superior glycaemic control versus metformin monotherapy due to a low rate of addition of antihyperglycemic agents. Diabetes Obes Metab. 2011;13:841-9.

9. Ali MK, Bullard KM, Saaddine JB, Cowie CC, Imperatore G, Gregg EW. Achievement of goals in U.S. diabetes care, 1999-2010. N Engl J Med. 2013;368:1613-24.

10. Inzucchi SE. Oral antihyperglycemic therapy for type 2 diabetes: scientific review. JAMA. 2002;287:360-72.

11. Amin NB, Wang X, Jain SM, Lee DS, Nucci G, Rusnak JM. Dose-ranging efficacy and safety study of ertugliflozin, a sodium-glucose co-transporter 2 inhibitor, in patients with type 2 diabetes on a background of metformin. Diabetes Obes Metab. 2015;17:591-8.

12. Amin NB, Wang X, Mitchell JR, Lee DS, Nucci G, Rusnak JM. Blood pressure-lowering effect of the sodium glucose co-transporter-2 inhibitor ertugliflozin, assessed via ambulatory blood pressure monitoring in patients with type 2 diabetes and hypertension. Diabetes Obes Metab. 2015;17:805-8.

13. Scheen AJ. Pharmacodynamics, efficacy and safety of sodium-glucose co-transporter type 2 (SGLT2) inhibitors for the treatment of type 2 diabetes mellitus. Drugs. 2015;75:33-59.

14. Drucker DJ, Nauck MA. The incretin system: glucagon-like peptide-1 receptor agonists and dipeptidyl peptidase- 4 inhibitors in type 2 diabetes. Lancet. 2006;368:1696-705.
15. Karasik A, Aschner P, Katzeff H, Davies MJ, Stein PP. Sitagliptin a DPP-4 inhibitor for the treatment of patients with type 2 diabetes: a review of recent clinical trials. Curr Med Res Opin. 2008;24:489-96.

16. Terra SG, Focht K, Davies M, Frias J, Derosa G, Darekar A, et al. Phase III, efficacy and safety study of ertugliflozin monotherapy in people with type 2 diabetes mellitus inadequately controlled with diet and exercise alone. Diabetes Obes Metab. 2017;19:721-8.

17. Rosenstock J, Frias J, Pall D, Charbonnel B, Pascu R, Saur D, et al. Effect of ertugliflozin on glucose control, body weight, blood pressure and bone density in type 2 diabetes mellitus inadequately controlled on metformin monotherapy (VERTIS MET). Diabetes Obes Metab. 2017. https://doi.org/ 10.1111/dom.13103.

18. Dagogo-Jack S, Liu J, Eldor R, Amorin G, Johnson J, Hille D, et al. Efficacy and safety of the addition of ertugliflozin in patients with type 2 diabetes mellitus inadequately controlled with metformin and sitagliptin: the VERTIS SITA2 placebo-controlled randomized study. Diabetes Obes Metab. 2017. https://doi.org/10.1111/dom.13116.

19. Eldor R, Pratley R, Golm G, et al. Effect of ertugliflozin plus sitagliptin on glycemic control versus either treatment alone in subjects with T2DM inadequately controlled with metformin (poster number 125-LB). In: 76th American Diabetes Association Scientific Sessions, 10-14 June 2016, New Orleans, LA, USA.

20. Cinti F, Moffa S, Impronta F, Cefalo CM, Sun VA, Sorice GP, et al. Spotlight on ertugliflozin and its potential in the treatment of type 2 diabetes: evidence to date. Drug Des Dev Ther. 2017;11:2905-19.

21. Raz I, Hanefeld M, Xu L, Caria C, Williams-Herman D, Khatami H. Efficacy and safety of the dipeptidyl peptidase-4 inhibitor sitagliptin as monotherapy in patients with type 2 diabetes mellitus. Diabetologia. 2006;49:2564-71.

22. Raz I, Chen $\mathrm{Y}, \mathrm{Wu}$ M, Hussain S, Kaufman KD, Amatruda JM, et al. Efficacy and safety of sitagliptin added to ongoing metformin therapy in patients with type 2 diabetes. Curr Med Res Opin. 2008;24:537-50.

23. U.S. Department of Health and Human Services. Guidance for industry diabetes mellitus: developing drugs and therapeutic biologics for treatment and prevention. 2008. https://www.fda.gov/downloads/ Drugs/.../Guidances/ucm071624.pdf. Accessed 6 Dec 2017. 
24. Tura A, Kautzky-Willer A, Pacini G. Insulinogenic indices from insulin and C-peptide: comparison of beta-cell function from OGTT and IVGTT. Diabetes Res Clin Pract. 2006;72:298-301.

25. American Diabetes Association. Executive summary: standards of medical care in diabetes-2012. Diabetes Care. 2012;35:S4-10.

26. University of Oxford. HOMA calculator. 2004. http://www.dtu.ox.ac.uk/homacalculator/. Accessed 6 Dec 2017.

27. Liang K-Y, Zeger SL. Longitudinal data analysis of continuous and discrete responses for pre-post designs. Sankhya Ser B. 2000;62:134-48.

28. Miettinen $\mathrm{O}$, Nurminen M. Comparative analysis of two rates. Stat Med. 1985;4:213-26.

29. American Diabetes Association. Standards of medical care in diabetes-2016. Diabetes Care. 2016;39:S1-112.

30. Lewin A, DeFronzo RA, Patel S, Liu D, Kaste R, Woerle $\mathrm{HJ}$, et al. Initial combination of empagliflozin and linagliptin in subjects with type 2 diabetes. Diabetes Care. 2015;38:394-402.

31. Merovci A, Solis-Herrera C, Daniele G, Eldor R, Fiorentino TV, Tripathy $\mathrm{D}$, et al. Dapagliflozin improves muscle insulin sensitivity but enhances endogenous glucose production. J Clin Invest. 2014;124:509-14.

32. Ferrannini E, Muscelli E, Frascerra S, Baldi S, Mari A, Heise T, et al. Metabolic response to sodium-glucose cotransporter 2 inhibition in type 2 diabetic patients. J Clin Invest. 2014;124:499-508.

33. Kalra S, Singh V, Nagrale D. Sodium-glucose cotransporter-2 inhibition and the glomerulus: a review. Adv Ther. 2016;33:1502-18.

34. Cherney DZ, Perkins BA, Soleymanlou N, Maione $\mathrm{M}$, Lai V, Lee A, et al. Renal hemodynamic effect of sodium-glucose cotransporter 2 inhibition in patients with type 1 diabetes mellitus. Circulation. 2014;129:587-97.

35. Fioretto P, Zambon A, Rossato M, Busetto L, Vettor R. SGLT2 inhibitors and the diabetic kidney. Diabetes Care. 2016;39(Suppl 2):S165-71.

36. Neal B, Perkovic V, Mahaffey KW, de Zeeuw D, Fulcher G, Erondu N, et al. Canagliflozin and cardiovascular and renal events in type 2 diabetes. N Engl J Med. 2017;377:644-57.

37. Wanner C, Inzucchi SE, Lachin JM, Fitchett D, von Eynatten M, Mattheus M, et al. Empagliflozin and progression of kidney disease in type 2 diabetes. N Engl J Med. 2016;375:323-34. 